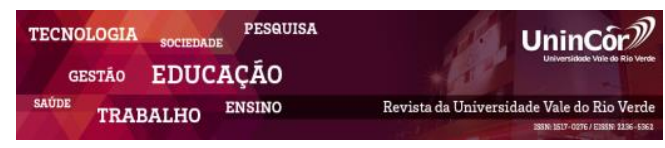

Revista da Universidade Vale do Rio Verde ISSN: 1517-0276 / EISSN: 2236-5362 v. 17 | n. 1 | Ano 2019

Bruna Aparecida dos Santos Universidade Estadual do Centro-Oeste bruna.apsantos97@gmail.com

Flávia Teixeira

Universidade Estadual do Centro-Oeste teixeiraflavia19@gmail.com

Luane Aparecida do Amaral Universidade Federal de Mato Grosso do Sul luapamaral@hotmail.com

Gabriela Arelhano Randolpho Universidade Federal de Mato Grosso do Sul gabrielaarelhano@yahoo.com.br

Kélin Schwarz

Universidade Federal do Triângulo Mineiro kelinschwarz@hotmail.com

Elisvânia Freitas dos Santos Universidade Federal de Mato Grosso do Sul elisvania@gmail.com

Juliano Tadeu Vilela de Resende Universidade Estadual do Centro-Oeste jvresende@uol.com

Daiana Novello Universidade Estadual do Centro-Oeste nutridai@gmail.com

\section{CARACTERIZAÇÃO QUÍMICA E NUTRICIONAL DE POLPA DE FRUTAS ARMAZENADAS SOB CONGELAMENTO}

\section{RESUMO}

O objetivo da pesquisa foi avaliar as características químicas e nutricionais de polpas de frutas in natura submetidas ao processo de congelamento. Foram analisadas as polpas de abacaxi, ameixa, caqui, laranja, maçã, mamão, maracujá, melão, morango e uva. As frutas foram congeladas a $-18{ }^{\circ} \mathrm{C}$ por um período de 60 dias e, em seguida foram realizadas análises da composição físico-química. Todas as frutas avaliadas foram classificadas como ácidas $(\mathrm{pH}<7)$. Os teores de sólidos solúveis totais variaram de $7{ }^{\circ}$ Brix na ameixa a $18{ }^{\circ}$ Brix no abacaxi. Menores teores de acidez titulável foram observados no caqui $(0,08 \%)$, laranja $(0,91 \%)$, mamão $(0,20 \%)$ e melão $(0,08 \%)$, enquanto maiores teores da relação sólidos solúveis totais/acidez titulável foram constatados para o caqui $(162,50)$ e para o melão $(125,00)$. $\mathrm{O}$ teor de umidade das frutas congeladas variou entre 85,03 g. $100 \mathrm{~g}^{-1}$ (maçã) a 93,29 g. $100 \mathrm{~g}^{-1}$ (ameixa). Houve pequena variação nos teores de cinzas $\left(0,26\right.$ a $\left.0,64 \mathrm{~g} \cdot 100 \mathrm{~g}^{-1}\right)$, proteína $\left(0,25\right.$ a 0,96 g. $\left.100 \mathrm{~g}^{-1}\right)$ e lipídio $\left(0,02\right.$ a $\left.0,37 \mathrm{~g} \cdot 100 \mathrm{~g}^{-1}\right)$. O teor de carboidrato foi menor na ameixa $\left(6 \mathrm{~g} .100 \mathrm{~g}^{-1}\right)$ e maior na maçã $\left(14,28 \mathrm{~g} \cdot 100 \mathrm{~g}^{-1}\right)$. Conclui-se que existem diferenças entre as características físico-químicas de polpa de frutas in natura submetidas ao processo de congelamento.

Palavras-chave: Análise de alimentos. Conservação. Frutas.

\section{CHEMICAL AND NUTRITIONAL CHARACTERIZATION OF FRUIT PULP STORED UNDER FREEZING}

\begin{abstract}
The objective of the research was to evaluate the chemical and nutritional characteristics of fresh fruit pulp submitted to the freezing process. The pulps of pineapple, plum, kaki, orange, apple, papaya, passion fruit, melon, strawberry and grape were analyzed. The fruits were frozen at $-18{ }^{\circ} \mathrm{C}$ for a period of 60 days and then physical-chemical composition analyzes were performed. All evaluated fruits were classified as acidic $(\mathrm{pH}<7)$. The total soluble solids contents ranged from $7^{\circ}$ Brix in the plum to $18^{\circ}$ Brix in the pineapple. Lower values of titratable acidity were observed in kaki $(0.08 \%)$, orange $(0.91 \%)$, papaya $(0.20 \%)$ and melon $(0.08 \%)$, while higher soluble solids/total acidity were found for kaki $(162,50)$ and melon $(125,00)$. The moisture content of frozen fruits ranged from 85.03 g. $100 \mathrm{~g}^{-1}$ (apple) to $93.29 \mathrm{~g} .100 \mathrm{~g}-1$ (plum). There was little variation in the

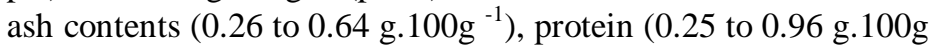
-1) and lipid (0.02 to $\left.0.37 \mathrm{~g} .100 \mathrm{~g}^{-1}\right)$. The carbohydrate content was lower in the plum $\left(6 \mathrm{~g} .100 \mathrm{~g}^{-1}\right)$ and higher in the apple $\left(14.28 \mathrm{~g} 100 \mathrm{~g}^{-1}\right)$. It is concluded that there are differences between the physico-chemical characteristics of fresh fruit pulp
\end{abstract}




\section{INTRODUÇÃO}

As frutas são alimentos indispensáveis na alimentação humana, uma vez que possuem um elevado teor nutricional. Geralmente, são compostas por nutrientes essenciais como as vitaminas, os minerais e as fibras, além do conteúdo expressivo de proteína, lipídio, carboidrato e outras substâncias essenciais. Dentre essas substâncias, destacam-se os antioxidantes naturais, que podem colaborar para a redução do estresse oxidativo nas células do organismo humano. $\mathrm{O}$ estresse oxidativo ocorre por acúmulo de moléculas de radicais livres, que são altamente instáveis e reagentes, causando dano celular (KUMAR et al., 2013). Nesse aspecto, o consumo de alimentos fonte de antioxidantes pode colaborar para a redução de processos inflamatórios, diminuindo o risco do desenvolvimento de doenças cardiovasculares, acidentes vasculares cerebrais e câncer (STIPANUK et al., 2013). Um exemplo de antioxidante presente em frutas e hortaliças é o ácido ascórbico, que auxilia na absorção do ferro dietético, no aumento da síntese hormonal e no fortalecimento do sistema imunológico (BRUNTON, 2012).

Após a colheita as frutas apresentam um shelf life bastante reduzido, o que acarreta em perdas nutricionais e econômicas. Além disso, há prejuízo para o meio ambiente, uma vez que se eleva o descarte de lixo orgânico. Uma alternativa para aumentar a disponibilidade das frutas é a preservação por congelamento, principalmente da polpa. As polpas de frutas são muito utilizadas pela indústria de alimentos na produção de diferentes produtos como geleias, bolos, sucos, sorvetes, dentre outros. Contudo, é possível que o processo de congelamento altere as características químicas e nutricionais desses alimentos. Menores teores de antocianinas e maior resposta da capacidade antioxidante foram verificados em polpa de caqui congelada, quando comparada à fruta in natura (MENDONÇA et al., 2015). Em morangos congelados houve aumento nos teores de ácido ascórbico e redução no teor de sólidos solúveis totais (SST) (ANDRADE JÚNIOR et al., 2016). Outra pesquisa demonstrou que para o maracujá, o congelamento reduziu o teor de SST, mas não afetou os valores de acidez titulável e de pH (RINALDI et al., 2017). Apesar da influência do congelamento, as características químicas e nutricionais de frutas e hortaliças podem ser afetadas também por outros fatores. Exemplos são o cultivar, o tempo de armazenamento sob congelamento, período de colheita e as condições climáticas durante a produção e o armazenamento (ANDRADE JÚNIOR et al., 2016). Nesse contexto, a presente pesquisa teve o objetivo de avaliar as características químicas e nutricionais de polpas de frutas in natura submetidas ao processo de congelamento.

\section{MATERIAL E MÉTODOS}




\subsection{Obtenção das amostras}

As frutas foram obtidas no comércio local do município de Guarapuava, PR. Foram analisadas as seguintes frutas: abacaxi (Ananas comosus), ameixa (Prunus domestica), caqui fuyu (Diospyros kaki), laranja pera (Citrus sinensis L.), maçã gala (Malus domestica), mamão formosa (Carica Papaya L.), maracujá (Passiflora edulis), melão (Cucumis melo), morango (Fragaria $x$ ananassa) e uva (Vitis vinífera). Todas as frutas foram, inicialmente, lavadas em água corrente. Em seguida, foram armazenadas em sacos plásticos escuros e congeladas a $-18{ }^{\circ} \mathrm{C}$ em freezer doméstico (Consul ${ }^{\circledR}$, Brasil), por um período de 60 dias.

\subsection{Composição química e nutricional}

Para as determinações analíticas, as frutas foram descongeladas em geladeira doméstica $\left(\right.$ Consul ${ }^{\circledR}$, Brasil $)$ em temperatura de $5^{\circ} \mathrm{C}\left( \pm 2{ }^{\circ} \mathrm{C}\right)$ por 24 horas. Na sequência, cinco polpas de cada fruta foram homogeneizadas individualmente em mixer doméstico (Britânia ${ }^{\circledR}$, Brasil). As análises foram realizadas em seguida a esse procedimento e em triplicata. Os resultados foram expressos em matéria úmida.

Foram realizadas as seguintes análises químicas: atividade de água (Aw), determinada utilizando-se o analisador de Aw (Novasina ${ }^{\circledR}$, Labswift model, Switzerland), operando com temperatura de $20{ }^{\circ} \mathrm{C} ; \mathrm{pH}$, que foi mensurado utilizando-se um pHmetro de bancada (Tecnopon ${ }^{\circledR}$, MPA-210 model, Brasil); sólidos solúveis totais (SST), que foi obtido pela leitura direta em refratômetro de bancada $\mathrm{ABBE}\left(\mathrm{Bel}^{\circledR}\right.$, modelo
RMI/RMT, Brasil) (AOAC, 2011). Os valores foram expressos em ${ }^{\circ}$ Brix. O método titulométrico (AOAC, 2011) foi utilizado para a determinação da acidez titulável (AT). Os resultados foram expressos em g de ácido cítrico/100. $\mathrm{g}^{-1}$ polpa. A relação SST/AT foi obtida por meio da divisão entre os valores de SST e AT. Os teores de açúcares redutores (AR), açúcares não redutores (ANR) e açúcares totais (AÇT), foram avaliados pelo método redutométrico de Lane-Eynon (AOAC, 2011). Os resultados foram expressos em g. $100 \mathrm{~g}^{-1}$.

$\mathrm{Na}$ análise da composição nutricional foram determinados os teores de umidade $\left(\right.$ g. $\left.100 \mathrm{~g}^{-1}\right)$; cinzas (g.100g $\left.\mathrm{g}^{-1}\right)$; proteína $\left(\mathrm{g} .100 \mathrm{~g}^{-1}\right)$ (AOAC, 2011); lipídio (g.100g ${ }^{-1}$ ) (BLIGH; DYER, 1959); carboidrato (g.100g $\left.\mathrm{g}^{-1}\right)$ por diferença, segundo a fórmula: \% Carboidratos $=100-(\%$ umidade + $\%$ proteína $+\%$ lipídio $+\%$ cinzas $+\%$ fibra $;$ valor energético total $\left(\mathrm{kcal} 100 \mathrm{~g}^{-1}\right)$, utilizando-se os valores recomendados por Atwater e Woods (1986) para lipídio (9 kcal/g), proteína (4 kcal/g) e carboidrato $(4 \mathrm{kcal} / \mathrm{g})$. O conteúdo de ácido ascórbico foi avaliado pelo método titulométrico padrão da AOAC (1984), modificado por Benassi e Antunes (1988). Os resultados foram expressos em $\mathrm{mg}$ de ácido ascórbico/100. $\mathrm{g}^{-1}$ polpa. A quantificação de antocianinas totais $\left(\mathrm{mg} .100 \mathrm{~g}^{-1}\right.$ ) foi realizada pelo método diferencial de $\mathrm{pH}$ (GIUSTI; WROSLTAD, 2001). As medidas de absorbância foram realizadas a $510 \mathrm{~nm}$ e $700 \mathrm{~nm}$ em espectrofotômetro (Agillent Technologies ${ }^{\circledR}$, Cary $60 \mathrm{UV}$, Malaysia). A atividade antioxidante foi avaliada pelo método ABTS (2,2-azinobis-[3etil-benzotiazolin-6-ácido sulfônico]), nas duas 
versões (hidrofílicas e lipofílicas) (MILLER et al., 1996). Os resultados foram expressos em mmol de equivalentes ao Trolox/g de amostra. A determinação dos compostos fenólicos foi realizada utilizando-se o método espectrofotométrico de FollinCiocauteau (BUCIĆ-KOJIĆ et al., 2007). A leitura foi realizada em espectrofotômetro (Agillent Technologies ${ }^{\circledR}$, Cary 60 UV, Malaysia) a $765 \mathrm{~nm}$. Os resultados foram expressos em mg de equivalente ácido gálico (GAE)/100.g $\mathrm{g}^{-1}$ polpa.

\subsection{Análise estatística}

Os resultados foram analisados por meio de análise de variância (ANOVA). As medias foram comparadas pelo teste de Scott-Knott com $5 \%$ de significância ( $\mathrm{p} \leq 0,05)$. O software Statistical Package for the Social Sciences (SPSS, Chicago, IL, USA) foi utilizado para a realização dos cálculos estatísticos.

\section{RESULTADOS E DISCUSSÃO}

\subsection{Composição química}

A composição química das polpas de frutas congeladas está apresentada na Tabela 1. Todas as frutas apresentaram elevada Aw (acima de 0,98$)$ e foram classificadas como ácidas $(\mathrm{pH}$ abaixo de 7), corroborando com a literatura (BUENO et al., 2002; CASTRO et al., 2015). Contudo, a ameixa, o abacaxi, a maçã, o maracujá, o morango e a uva foram aquelas com maior acidez $(\mathrm{pH}<4)$. O maracujá, a ameixa, o morango e o abacaxi apresentaram os maiores teores de $\operatorname{AT}(\geq 6,0)$, enquanto o caqui, o melão, o mamão e a laranja foram as frutas com menor AT $(\leq 1,00)$. Uma variação de ácido cítrico nas frutas entre $0,08 \%$ a $1,95 \%$ implica em um sabor suave com boa aceitação pelo consumidor (PAIVA et al., 1997). Além disso, alguns ácidos orgânicos presentes nas frutas são voláteis, o que contribui para um aroma mais agradável (CHITARRA; CHITARRA, 2005).

Os SST incluem importantes compostos responsáveis pelo sabor das frutas (KLUGE et al., 2002; PORTELA et al., 2012). Exemplos são os açúcares solúveis e, em alguns casos, o teor de aminoácidos, ácidos orgânicos, vitaminas e algumas pectinas. Dessa forma, quanto maior o teor de SST na fruta, maior a concentração desses compostos (CHITARRA; CHITARRA, 2005). Na presente pesquisa, os teores de SST variaram de 7 ${ }^{\circ}$ Brix na ameixa a $18^{\circ}$ Brix no abacaxi. Apesar dos valores de SST representarem uma importante avaliação do teor de açúcares nas frutas, a relação SST/AT mostra-se eficiente para demonstrar o grau de maturação e sabor do fruto (CHITARRA; CHITARRA, 2005). Durante o processo metabólico do amadurecimento das frutas, os ácidos orgânicos são degradados, uma vez que há um aumento da concentração de enzimas hidrolases, peroxidases e catalase, o que reduz o sabor ácido da fruta. Dessa forma, a relação SST/AT se eleva, aumentando também a doçura do produto e sua aceitabilidade (PEREIRA et al., 2006). Maior relação SST/AT foi observada no caqui, no mamão e no melão. O teor de SST em frutas pode aumentar durante o congelamento, devido à degradação da parede celular e da ruptura das membranas (POIANA et al., 2010; VEBERIC et al., 2014). 
Os valores de AR (glicose e frutose) variaram de 2,38 g. $100 \mathrm{~g}^{-1}$ na laranja a 12,31 g. $100 \mathrm{~g}^{-1}$ no caqui, enquanto os ANR (sacarose) variaram entre 0,64 g. $100 \mathrm{~g}^{-1}$ na maçã a 12,05 g. $100 \mathrm{~g}^{-1}$ no abacaxi. Os teores AÇT variaram entre 4,96 g. $100 \mathrm{~g}^{-1}$ na laranja e 16,67 no abacaxi g. $100 \mathrm{~g}^{-1}$. Teores inferiores de AÇT para polpa de uva congelada $\left(9,56 \mathrm{~g} \cdot 100 \mathrm{~g}^{-1}\right)$ e superiores para o maracujá $\left(11,37\right.$ g. $\left.100 \mathrm{~g}^{-1}\right)$ foram observados em outros estudos (SALGADO et al., 1999; SOUZA et al., 2012). A frutose é quem possui maior poder adoçante, dentre os AR presentes na fruta, o que pode melhorar a aceitabilidade sensorial do alimento (CHITARRA; CHITARRA, 2005). Dessa forma, destacam-se na presente pesquisa o morango, a maçã, a uva e o caqui que apresentaram teores de AR mais elevados (acima de $\left.6 \mathrm{~g} \cdot 100 \mathrm{~g}^{-1}\right)$. No congelamento, o teor de AR pode aumentar devido à degradação da sacarose, que é convertida em glicose e frutose. Contudo, os teores de ANR podem reduzir devido ao processo respiratório normal da fruta (DAMIANI et al., 2013; DUARTE et al., 2017).

Apenas o mamão atendeu à legislação brasileira (BRASIL, 2000) para os parâmetros de SST, pH, AT e AÇT. A uva obteve menor teor de SST quando comparada ao disposto na legislação (14 ํix). Já o maracujá, apresentou menor valor de $\mathrm{pH}$ que o recomendado (mínimo de 2,7). As demais frutas ameixa, abacaxi, caqui, laranja, maçã, morango não possuem valores estabelecidos na legislação para os parâmetros avaliados.

\subsection{Composição nutricional}

Em relação à composição nutricional (Tabela 2), o teor de umidade das frutas congeladas variou de 85,03 g. $100 \mathrm{~g}^{-1}$ na maçã a 93,29 g.100g ${ }^{-1}$ na ameixa. Resultados similares foram observados por Souza et al. (2012), que estudaram a polpa do maracujá, que apresentou um teor de umidade médio de $84,12 \mathrm{~g} \cdot 100 \mathrm{~g}^{-1}$. Esse alto teor de umidade é característico de frutas, já que elas apresentam alta Aw. Assim, as frutas possuem um shelf-life reduzido, já que são altamente suscetíveis à ação microbiana, que atua mais rapidamente em alimentos com Aw entre 0,60 a 0,98 (FRANCO; LANDGRAF, 2005). Houve pequena variação nos teores de cinzas $(0,26$ a 0,64 g. $\left.100 \mathrm{~g}^{-1}\right)$, proteína $\left(0,25\right.$ a 0,96 g. $\left.100 \mathrm{~g}^{-1}\right)$ e lipídio $\left(0,02\right.$ a $\left.0,37 \mathrm{~g} \cdot 100 \mathrm{~g}^{-1}\right)$. Além disso, foi possível constatar que o teor desses nutrientes em frutas são baixos $\left(\leq 1 \mathrm{~g} \cdot 100 \mathrm{~g}^{-1}\right)$. Resultados similares foram observados em polpa congelada de maracujá (SOUZA et al., 2012; SPADA et al., 2008) e outras como maçã, melão, laranja, abacaxi e morango (SPADA et al., 2008). Contudo, as frutas são importantes fontes de carboidrato, vitaminas, minerais, fibra, compostos fenólicos e antocianinas. Esses compostos devem ser consumidos em uma dieta habitual, já que podem reduzir o risco de doenças crônicas não transmissíveis (DCNT) como a hipertensão arterial e a aterosclerose (RAFIEIAN-KOPAEI et al., 2016; SEDIGHI et al., 2017).

$\mathrm{O}$ teor de carboidrato variou de $6 \mathrm{~g} \cdot 100 \mathrm{~g}^{-1}$ na ameixa a 14,28 g. $100 \mathrm{~g}^{-1}$ na maçã. Consequentemente, o valor calórico foi menor na ameixa $\left(26,10\right.$ g. $\left.100 \mathrm{~g}^{-1}\right)$ e maior na maçã $(58,56$ g. $\left.100 \mathrm{~g}^{-1}\right)$. Apesar das frutas apresentarem um teor 
de carboidrato elevado, parte desse nutriente é composto por fibra (ARAÚJO et al., 2014). A fibra apresenta um importante papel na saúde, já que melhora o trânsito intestinal, aumentando a formação do bolo fecal, o que auxilia na redução dos níveis de lipoproteína de baixa densidade (LDL), de colesterol total e da glicemia pósprandial (FULLER et al., 2016). Dessa forma, o 
Tabela 1. Composição química de polpa de frutas in natura submetidas ao processo de congelamento

\begin{tabular}{|c|c|c|c|c|c|c|c|c|c|c|}
\hline Parâmetro & Abacaxi & Ameixa & Caqui & Laranja & Maçã & Mamão & Maracujá & Melão & Morango & Uva \\
\hline Aw & $0,99 \pm 0,00^{\mathrm{a}}$ & $0,99 \pm 0,00^{\mathrm{a}}$ & $0,98 \pm 0,00^{b}$ & $0,99 \pm 0,00^{\mathrm{a}}$ & $0,98 \pm 0,00^{\mathrm{b}}$ & $0,98 \pm 0,00^{b}$ & $0,98 \pm 0,00^{\mathrm{b}}$ & $0,99 \pm 0,00^{\mathrm{a}}$ & $0,98 \pm 0,00^{\mathrm{b}}$ & $0,98 \pm 0,00^{\mathrm{b}}$ \\
\hline $\mathrm{pH}$ & $3,59 \pm 0,01^{\mathrm{f}}$ & $3,07 \pm 0,01^{\mathrm{i}}$ & $5,28 \pm 0,01^{\mathrm{b}}$ & $4,06 \pm 0,01^{\mathrm{d}}$ & $3,88 \pm 0,01^{\mathrm{e}}$ & $4,50 \pm 0,01^{\mathrm{c}}$ & $2,61 \pm 0,01^{j}$ & $6,00 \pm 0,01^{\mathrm{a}}$ & $3,35 \pm 0,01^{\mathrm{h}}$ & $3,50 \pm 0,00^{\mathrm{g}}$ \\
\hline Acidez Titulável (\% ácido cítrico) & $6,00 \pm 0,01^{\mathrm{d}}$ & $11,30 \pm 0,00^{\mathrm{b}}$ & $0,08 \pm 0,00^{\mathrm{i}}$ & $0,91 \pm 0,01^{\mathrm{g}}$ & $1,47 \pm 0,01^{\mathrm{f}}$ & $0,20 \pm 0,01^{\mathrm{h}}$ & $39,13 \pm 0,02^{\mathrm{a}}$ & $0,08 \pm 0,00^{\mathrm{i}}$ & $6,13 \pm 0,02^{\mathrm{c}}$ & $3,57 \pm 0,00^{\mathrm{e}}$ \\
\hline Sólidos Solúveis Totais ( ${ }^{\circ}$ Brix) & $18,00 \pm 0,00^{\mathrm{a}}$ & $7,00 \pm 0,00^{g}$ & $13,00 \pm 0,00^{\mathrm{b}}$ & $11,00 \pm 0,00^{\mathrm{d}}$ & $13,00 \pm 0,00^{\mathrm{b}}$ & $11,00 \pm 0,00^{\mathrm{d}}$ & $12,00 \pm 0,00^{\mathrm{c}}$ & $10,00 \pm 0,00^{\mathrm{e}}$ & $8,00 \pm 0,00^{f}$ & $11,00 \pm 0,00^{\mathrm{d}}$ \\
\hline Relação Sólidos Solúveis Totais/Acidez titulável & $3,00 \pm 0,00^{\mathrm{g}}$ & $0,62 \pm 0,01^{\mathrm{i}}$ & $162,50 \pm 0,00^{\mathrm{a}}$ & $12,13 \pm 0,08^{\mathrm{d}}$ & $9,23 \pm 0,10^{\mathrm{e}}$ & $54,97 \pm 0,06^{\mathrm{c}}$ & $0,31 \pm 0,00^{\mathrm{j}}$ & $125,00 \pm 0,00^{\mathrm{b}}$ & $1,30 \pm 0,02^{\mathrm{h}}$ & $3,09 \pm 0,05^{\mathrm{f}}$ \\
\hline Açúcares Redutores (g.100g $\left.{ }^{-1}\right)$ & $4,61 \pm 0,01^{\mathrm{g}}$ & $5,87 \pm 0,02^{\mathrm{e}}$ & $12,31 \pm 0,02^{\mathrm{a}}$ & $2,38 \pm 0,01^{\mathrm{i}}$ & $9,01 \pm 0,00^{c}$ & $5,86 \pm 0,02^{\mathrm{e}}$ & $2,97 \pm 0,01^{\mathrm{h}}$ & $5,12 \pm 0,01^{\mathrm{f}}$ & $6,59 \pm 0,02^{\mathrm{d}}$ & $10,37 \pm 0,07^{\mathrm{b}}$ \\
\hline Açúcares Não Redutores (g. $\left.100 \mathrm{~g}^{-1}\right)$ & $12,05 \pm 0,01^{\mathrm{a}}$ & $1,72 \pm 0,03^{\mathrm{h}}$ & $3,22 \pm 0,05^{\mathrm{d}}$ & $2,60 \pm 0,01^{\mathrm{f}}$ & $0,64 \pm 0,05^{\mathrm{i}}$ & $2,43 \pm 0,07^{\mathrm{g}}$ & $4,03 \pm 0,06^{\mathrm{c}}$ & $5,66 \pm 0,06^{\mathrm{b}}$ & $3,23 \pm 0,08^{\mathrm{d}}$ & $2,98 \pm 0,05^{\mathrm{e}}$ \\
\hline Açúcares Totais $\left(\mathrm{g} .100 \mathrm{~g}^{-1}\right)$ & $16,67 \pm 0,03^{\mathrm{a}}$ & $7,60 \pm 0,03^{\mathrm{h}}$ & $15,82 \pm 0,04^{\mathrm{b}}$ & $4,96 \pm 0,01^{\mathrm{j}}$ & $9,65 \pm 0,05^{\mathrm{f}}$ & $8,29 \pm 0,08^{\mathrm{g}}$ & $6,99 \pm 0,05^{\mathrm{i}}$ & $10,78 \pm 0,07^{\mathrm{d}}$ & $9,83 \pm 0,05^{\mathrm{e}}$ & $13,39 \pm 0,10^{\mathrm{c}}$ \\
\hline
\end{tabular}

Letras distintas na linha indicam diferença significativa pelo teste de Scott-Knott $(\mathrm{p}<0,05)$. Resultados apresentados em base úmida. Fonte: os autores.

Tabela 2. Composição nutricional de polpa de frutas in natura submetidas ao processo de congelamento

\begin{tabular}{|c|c|c|c|c|c|c|c|c|c|c|}
\hline Parâmetro & Abacaxi & Ameixa & Caqui & Laranja & Maçã & Mamão & Maracujá & Melão & Morango & Uva \\
\hline$\overline{\mathrm{U}}$ & & & & & & & & & & \\
\hline $\mathrm{C}$ & & & $0^{\mathrm{f}}$ & & & & & & & \\
\hline $\left..100 \mathrm{~g}^{-1}\right)$ & $0,45 \pm 0,02^{\mathrm{e}}$ & $0,39 \pm 0,01^{\mathrm{f}}$ & $0,30 \pm 0,01^{\mathrm{g}}$ & $0,83 \pm 0,02^{b}$ & $0,25 \pm 0,01^{\mathrm{h}}$ & $0,47 \pm 0,00^{\mathrm{e}}$ & $0,96 \pm 0,02^{\mathrm{a}}$ & $0,50 \pm 0,01^{\mathrm{e}}$ & $0,77 \pm 0,01^{\mathrm{c}}$ & $0,62 \pm 0,01^{\mathrm{d}}$ \\
\hline & d & $0,06 \pm 0,00^{f}$ & $0,04 \pm 0,00^{f}$ & $0,13 \pm 0,00^{c}$ & $0,05 \pm 0,00^{\mathrm{f}}$ & $0,12 \pm 0,01^{\mathrm{d}}$ & $0,37 \pm 0,02^{\mathrm{a}}$ & $0,02 \pm 0,00^{\mathrm{f}}$ & $0,27 \pm 0,00^{\mathrm{b}}$ & $0,08 \pm 0,01^{\mathrm{e}}$ \\
\hline $\left.00 \mathrm{~g}^{-1}\right)$ & $0^{c}$ & $6^{\mathrm{i}}$ & $0^{\mathrm{b}}$ & 9,47 & $14,28 \pm 0,09^{\mathrm{a}}$ & 9,4 & 11 , & $6,48 \pm 0,02^{\mathrm{h}}$ & $0,04^{\mathrm{g}}$ & $0,02^{\mathrm{e}}$ \\
\hline $\left.100 g^{-1}\right)$ & 53,12 & $30^{1}$ & $4^{b}$ & $42,38 \pm 0,27^{\mathrm{e}}$ & $58,56 \pm 0,32^{\mathrm{a}}$ & $40,58 \pm 0,05^{\mathrm{f}}$ & $52,54 \pm 0,56^{\mathrm{c}}$ & $28,13 \pm 0,11^{\mathrm{h}}$ & $35,71 \pm 0,24^{\mathrm{g}}$ & $47,90 \pm 0,09^{\mathrm{d}}$ \\
\hline & $35,95 \pm 0,02^{\mathrm{b}}$ & 20,46 & $88,26 \pm 0,01^{\mathrm{f}}$ & $365,14 \pm 0,01^{\mathrm{a}}$ & $54,49 \pm 0,01^{\mathrm{g}}$ & $308,72 \pm 0,01^{\mathrm{c}}$ & $164,38 \pm 0,01^{\mathrm{d}}$ & $29,45 \pm 0,01^{\mathrm{i}}$ & $90,18 \pm 0,01^{\mathrm{e}}$ & $32,82 \pm 0,01^{\mathrm{h}}$ \\
\hline An & $*$ & & $0,39 \pm 0,04^{\mathrm{d}}$ & $*$ & $0,42 \pm 0,03^{\mathrm{d}}$ & $0,84 \pm 0,06^{\mathrm{b}}$ & $0,06 \pm 0,01^{\mathrm{e}}$ & $0,43=$ & $14,11 \pm 0,06^{\mathrm{a}}$ & $0,74 \pm 0,03^{\mathrm{c}}$ \\
\hline Compostos Fenólicos (mg GAE.100g ${ }^{-1}$ ) & $5,56 \pm 0,01^{\mathrm{i}}$ & $14,15 \pm 0,01^{\mathrm{d}}$ & $3,73 \pm 0,06^{\mathrm{j}}$ & $14,57 \pm 0,03^{\mathrm{c}}$ & $10,19 \pm 0,01^{\mathrm{g}}$ & $14,84 \pm 0,00^{\mathrm{b}}$ & $20,18 \pm 0,03^{\mathrm{a}}$ & $10,76 \pm 0,00^{\mathrm{f}}$ & $9,79 \pm 0,03^{\mathrm{h}}$ & $14,04 \pm 0,03^{\mathrm{e}}$ \\
\hline
\end{tabular}

Letras distintas na linha indicam diferença significativa pelo teste de Scott-Knott $(\mathrm{p}<0,05)$. Resultados apresentados em base úmida. Fonte: os autores. *Não detectado. 
consumo adequado de fibra pode reduzir o risco de doenças como o câncer colorretal e de DCNT como a diabetes mellitus tipo 2 (THREAPLETON et al., 2013; BRADBURY et al., 2014; FULLER et al., 2016). De forma geral, os teores de macronutrientes são pouco influenciados pelo processo de congelamento (ORDÓÑEZ, 2005).

Houve uma variação significativa nos valores de ácido ascórbico das frutas (20,46 mg. $100 \mathrm{~g}^{-1}$ na ameixa a $365,14 \mathrm{mg} \cdot 100 \mathrm{~g}^{-1}$ na laranja). A laranja, o abacaxi, o mamão e o maracujá foram os que apresentaram as quantidades mais significativas de vitamina $\mathrm{C}$ (acima de $100 \mathrm{mg} \cdot 100 \mathrm{~g}^{-1}$ ). Já a uva, o melão e a ameixa tiveram a menor concentração (abaixo de $\left.40 \mathrm{mg} .100 \mathrm{~g}^{-1}\right)$, corroborando com a literatura (SPADA et al., 2008; SOUZA et al., 2014). No organismo humano, a vitamina $\mathrm{C}$ atua no metabolismo do ferro, aumentando sua biodisponibilidade, além de participar da biossíntese de colágeno (VANNUCCHI; ROCHA, 2012). Nesse contexto, o consumo diário aproximado de $27 \mathrm{~g}$ de abacaxi, $440 \mathrm{~g}$ de ameixa, $102 \mathrm{~g}$ de caqui, $25 \mathrm{~g}$ de laranja, $165 \mathrm{~g}$ maçã, $29 \mathrm{~g}$ mamão, 55 g de maracujá, 306 g de melão, 100 g de morango e $274 \mathrm{~g}$ de uva atingiria a recomendação diária média de vitamina $\mathrm{C}$ para um homem adulto (90 mg/dia) (IOM, 2000). Por ser uma vitamina com elevado grau de oxidação, o congelamento pode reduzir de forma significativa o seu conteúdo em polpas de frutas, como já descrito por outros autores (BRUNINI et al., 2002; BARANKEVICZ et al., 2015).

As antocianinas fazem parte dos pigmentos polifenólicos, presentes em frutas e hortaliças, variando da cor vermelha ao laranja e do azul ao violeta (WALLACE; GIUSTI, 2015). Pesquisas demonstraram que o consumo de antocianinas pode reduzir o risco infarto do miocárdio (CASSIDY et al., 2013, PASCUALTERESA, 2014). Além disso, as antocianinas possuem efeito neuroprotetor na doença de Parkinson (STRATHEARN et al., 2014) e auxiliam no tratamento de pacientes com hiperlipidemias (VALENCIA, 2017). No presente estudo, apenas o morango apresentou um teor mais significativo de antocianinas $\left(14,11 \mathrm{mg} \cdot 100 \mathrm{~g}^{-1}\right)$. As demais frutas tiveram valores abaixo de 1 mg. $100 \mathrm{~g}^{-1}$, com destaque para o maracujá, que apresentou a menor concentração $\left(0,06 \mathrm{mg} \cdot 100 \mathrm{~g}^{-}\right.$ ${ }^{1}$ ), corroborando com avaliações de Kuskoski et al. (2006). Esses resultados ocorrem devido ao morango apresentar uma coloração avermelhada, o que em geral representa uma maior concentração de antocianinas. Além disso, o morango possui um pH ácido, o que promove uma maior estabilidade desse composto (DELGADO-VARGAS et al., 2000). Pesquisas demonstraram que as antocianinas são pouco afetadas pelo congelamento (LOHACHOOMPOL et al., 2004; ARAÚJO et al., 2009), já que sua degradação está associada à temperaturas mais elevadas, acima de $50{ }^{\circ} \mathrm{C}$ (PATRAS et al., 2010).

Maior teor de compostos fenólicos $(\mathrm{p}<0,05)$ foi verificado na polpa de maracujá. Contudo, a ameixa, a laranja, o mamão, o melão e a uva também se destacaram, já que apresentaram um teor de fenólicos superior a $10 \mathrm{mg}$ GAE. $100 \mathrm{~g}^{-1}$. $\mathrm{O}$ caqui foi a fruta com menor concentração de fenólicos. Os resultados do presente estudo são 
superiores aos encontrados na literatura (SPADA et al., 2008) para polpas congeladas de maçã, melão, laranja, mamão, maracujá, abacaxi e morango. $\mathrm{O}$ processo de congelamento pode aumentar ou reduzir o teor de compostos fenólicos. Essas substâncias podem sofrer reações de oxidação durante o congelamento, pela ação das enzimas peroxidase e a polifenoloxidase, o que reduz o seu conteúdo em frutas. Por outro lado, a fenilalanina amônia-liase, principal enzima no metabolismo secundário dos compostos fenólicos, atua no metabolismo do ácido chíquimico. A fenilalanina é convertida em ácido cinâmico, gerando novos compostos fenólicos, fato que pode elevar sua concentração nas frutas (DING et al., 2001; LIMA et al., 2012).

Assim como o organismo humano se utiliza de mecanismos endógenos para se proteger da ação dos radicais livres (BARREIROS et al., 2006), as plantas também possuem esse processo. Os compostos fenólicos e as antocianinas são as principais substâncias com efeito antioxidante presentes nas frutas (KALT et al., 1999). Na Figura 1 estão apresentados os resultados da capacidade antioxidante das polpas das frutas. As polpas de melão e de laranja apresentaram os teores de capacidade antioxidante hidrofílica e lipofílica mais baixos $\left(<\right.$ que $1.400 \mu \mathrm{mol} / 100 \mathrm{~g}^{-1}$, $\mathrm{p}<0,05)$. As demais frutas tiveram pequenas variações nesse parâmetro (média de 1.519,88 $\mu$ mol.100g $\mathrm{g}^{-1}$ para hidrofílica e $1.530,59$ $\mu$ mol.100g $\mathrm{g}^{-1}$ para lipofílica).

Capacidade antioxidante hidrofílica

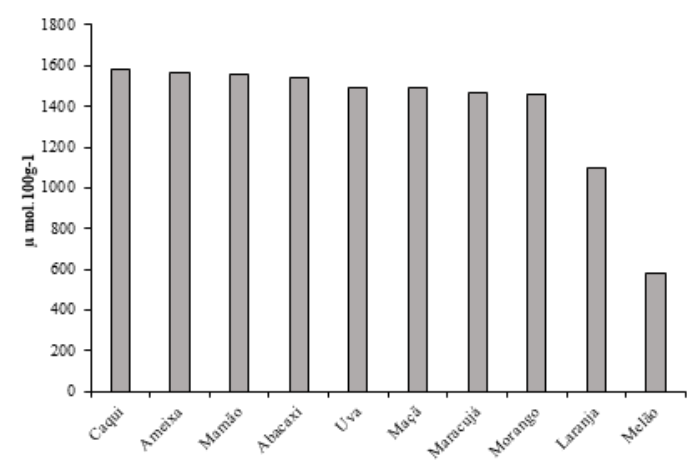

Capacidade antioxidante lipofílica

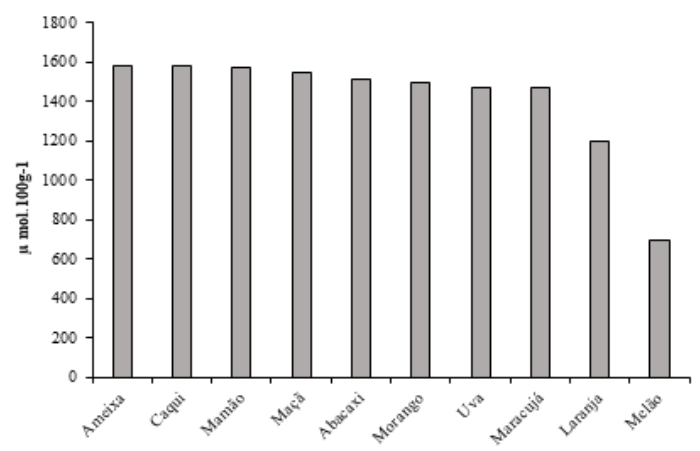

Letras distintas na linha indicam diferenças significativas pelo teste de Scott-Knott $(\mathrm{p}<0,05)$. Resultados reportados em base úmido. Fonte: os autores.

Figura 1. Capacidade antioxidante hidrofílica e lipofílica de polpa de frutas in natura submetidas ao processo de congelamento.

\section{CONCLUSÃO}

Conclui-se que existem diferenças entre as características químicas e nutricionais de polpa de frutas in natura submetidas ao processo de congelamento. Contudo, parâmetros como acidez titulável, relação sólidos solúveis/acidez titulável, vitamina $\mathrm{C}$, antocianinas, compostos fenólicos e capacidade antioxidante são aqueles que apresentam maior variação entre as frutas avaliadas. Sugere-se a atualização da legislação brasileira referente à regulamentação dos padrões 
de polpa de frutas, já que o documento vigente inclui um número restrito de informações.

\section{AGRADECIMENTOS}

Os autores agradecem à Fundação Araucária de Apoio ao Desenvolvimento Científico e Tecnológico do Estado do Paraná, Brasil pela concessão de bolsa de estudos de iniciação científica.

\section{REFERÊNCIAS}

ANDRADE JÚNIOR, V. C.; GUIMARÃES, A. G.; AZEVEDO, A. M.; PINTO, N. A. V. D.; FERREIRA, M. A. M. Conservação pós-colheita de frutos de morangueiro em diferentes condições de armazenamento. Horticultura Brasileira, v. 34, n. 3, p.405-411, 2016.

ASSOCIATION OF OFFICIAL ANALYTICAL Chemists (AOAC). Official methods of analysis of AOAC International. 14th ed. Gaithersburg: AOAC, 1984.

ASSOCIATION OF OFFICIAL ANALYTICAL Chemists (AOAC). Official methods of analysis of AOAC. 17th ed. Gaithersburg: AOAC, 2011.

ARAÚJO, P. F.; RODRIGUES, R. D. S.; MACHADO, A. R., SANTOS, V. D. S.; SILVA, J. A. Influência do congelamento sobre as características físico-químicas e o potencial antioxidante de néctar de amora-preta. Boletim do Centro de Pesquisa de Processamento de Alimentos, v. 27, n. 2, p. 199-206, 2009.

ARAÚJO, W. M. C.; MONTEBELLO, N. P.; BOTELHO, R. B. A. Alquimia dos AlimentosSérie Alimentos e Bebidas. Brasília: Senac, 2014.

ATWATER, W. O.; WOODS, C. D. The chemical composition of American food materials. Washington: Government Printing Office, 1896.

BARANKEVICZ, G. B.; NOVELLO, D.; RESENDE, J. T. V.; SCHWARZ, K.; SANTOS, E. F. Características físico-químicas de amostras congeladas de híbridos de tomateiro para processamento. Horticultura Brasileira, v. 33, n. 1, p 7-11, 2015.

BARREIROS, A. L. B. S.; DAVID, J. M.; DAVID, J. P. D. L. Estresse oxidativo: relação entre geração de espécies reativas e defesa do organismo. Química Nova, v. 29, n. 1, p. 113123, 2006.

BENASSI, M. T.; ANTUNES, A. J. A comparison of metaphosphoric and oxalic acids as extractans solutions for the determination of vitamin $C$ in selected vegetables. Arquivos de Biologia e Tecnologia, v. 31, n. 4, p. 507-513, 1988.

BLIGH, E. G.; DYER, W. J. A rapid method of total lipid extraction and purification. Canadian journal of biochemistry and physiology, v. 37, n. 8, p. 911-917, 1959.

BRADBURY, K. E.; APPLEBY, P. N.; KEY, T. $J$. Fruit, vegetable, and fiber intake in relation to cancer risk: findings from the European Prospective Investigation into Cancer and Nutrition (EPIC). The American Journal of Clinical Nutrition, v. 100, n. suppl_1, p. 394S398S, 2014.

BRASIL. Ministério da Agricultura, Pecuária e Abastecimento. MAPA. IN n ${ }^{\circ} 01$, de 07 de janeiro de 2000. Regulamento Técnico Geral para Fixação dos Padrões de Identidade e Qualidade para Polpa de Fruta. Diário Oficial da República Federativa do Brasil, Brasília, DF, 10 jan. 2000.

BRUNINI, M. A.; DURIGAN, J. F.; OLIVEIRA, A. L. Avaliação das alterações em polpa de manga 'Tommy-Atkins' congeladas. Revista Brasileira de Fruticultura, v. 24, n. 3, p. 651-653, 2002.

BRUNTON, L. As bases farmacológicas da terapêutica de Goodman e Gilman. 12. ed. Porto Alegre: McGraw Hill, 2012.

BUCIĆ-KOJIĆ, A.; PLANINIĆ, M.; TOMAS, S.; BILIĆ, M.; VELIĆ, D. Study of solid-liquid extraction kinetics of total polyphenols from grape seeds. Journal of Food Engineering, v. 81, n. 1, p. 236-242, 2007.

BUENO, S. M.; LOPES, M. D. R. V.; GRACIANO, R. A.; FERNANDES, E. C.; GARCIA-CRUZ, C. H. Avaliação da qualidade de 
polpas de frutas congeladas. Revista do Instituto Adolfo Lutz, v. 62, n. 2, p. 121-126, 2002.

CASSIDY, A.; MUKAMAL, K. J.; LIU, L.; FRANZ, M.; ELIASSEN, A. H.; RIMM, E. B. High Anthocyanin Intake Is Associated With a Reduced Risk of Myocardial Infarction in Young and Middle-Aged Women Clinical Perspective. Circulation, v. 127, n. 2, p. 188-196, 2013.

CASTRO, T. M. N.; ZAMBONI, P. V.; DOVADONI, S.; CUNHA NETO, A.; RODRIGUES, L. J. Parameters of quality of frozen fruit. Revista do Instituto Adolfo Lutz, v. 74, n. 4, p. 426-436, 2015.

CHITARRA, M. I. F.; CHITARRA, A. B. Póscolheita de frutos e hortaliças: fisiologia $e$ manuseio. 2. ed. Lavras: UFLA, 2005.

DAMIANI, C.; LAGE, M. E.; SILVA, F. A. D.; PEREIRA, D. E. P.; BECKER, F. S. Changes in the physicochemical and microbiological properties of frozen araça pulp during storage. Food Science and Technology, v. 33, n. 1, p. 19-27, 2013.

DELGADO-VARGAS, F.; JIMÉNEZ, A. R.; PAREDES-LÓPEZ, O. Natural pigments: carotenoids, anthocyanins, and betalainscharacteristics, biosynthesis, processing, and stability. Critical Reviews in Food Science and Nutrition, v. 40, n. 3, p. 173-289, 2000.

DING, C. K.; CHACHIN, K.; UEDA, Y.; IMAHORI, Y.; WANG, C. Y. Metabolism of phenolic compounds during loquat fruit development. Journal of Agricultural and Food Chemistry, v. 49, n. 6, p. 2883-2888, 2001.

DUARTE, M. H.; QUEIROZ, E. D. R.; ROCHA, D. A.; COSTA, A. C.; ABREU, C. M. P. D. Quality of pitaya (Hylocereus undatus) submitted to organic fertilization and stored under refrigeration. Brazilian Journal of Food Technology, v. 20, e2015115, 2017.

FRANCO, B. D. G. M.; LANDGRAF, M. Microbiologia dos alimentos. São Paulo: Atheneu, 2005.

FULLER, S.; BECK, E.; SALMAN, H.; TAPSELL, L. New horizons for the study of dietary fiber and health: a review. Plant Foods For Human Nutrition, v. 71, n. 1, p. 1-12, 2016.
GIUSTI, M. M.; WROLSTAD, R. E.

Anthocyanins. Characterization and Measurement with UV-Visible Spectroscopy. In: WROLSTAD, R. E. (Ed.). Current Protocols in Food Analytical Chemistry. New York: John Wiley \& Sons, 2001.

INSTITUTE OF MEDICINE (IOM). Dietary reference intakes for vitamin $C$, vitamin $E$, selenium, and carotenoids. Washington: National Academy Press, 2000.

KALT, W.; FORNEY, C. F.; MARTIN, A.; PRIOR, R. L. Antioxidant capacity, vitamin C, phenolics, and anthocyanins after fresh storage of small fruits. Journal of Agricultural and Food Chemistry, v. 47, n. 11, p. 4638-4644, 1999.

KLUGE, R. A.; NACHTIGAL, J. C.; FACHINELLO, J. C.; BILHALVA, A. $B$. Fisiologia e manejo pós-colheita de frutas de clima temperado. São Paulo: Editora Rural, 2002.

KUMAR, V.; ABBAS, A. K.; ASTER, J. C. Robbins: Patologia Básica. 9. ed. Rio de Janeiro: Elsevier, 2013.

KUSKOSKI, E. M.; ASUERO, A. G.; MORALES, M. T.; FETT, R. Frutos tropicais silvestres e polpas de frutas congeladas: atividade antioxidante, polifenóis e antocianinas. Ciência Rural, v. 36, n. 4, p. 1283-1287, 2006.

LIMA, R. S. N.; OLIVEIRA, J. T. A.; AZEVEDO MOREIRA, R. Diferenças Genômicas entre plantas e suscetíveis a doenças: Técnicas Disponíveis. Revista Brasileira em Promoção da Saúde, v. 14, n. 1, p. 44-50, 2012.

LOHACHOOMPOL, V.; SRZEDNICKI, G.; CRASKE, J. The change of total anthocyanins in blueberries and their antioxidant effect after drying and freezing. BioMed Research International, v. 2004, n. 5, p. 248-252, 2004.

MENDONÇA, V. Z.; DAIUTO, E. R.; FURLANETO, K. A.; RAMOS, J. A.; FUJITA, E.; VIEITES, R. L.; TECCHIO, M. A.; CARVALHO, L. R. Aspectos físico-químicos e bioquímicos durante o armazenamento refrigerado do caqui em atmosfera modificada passiva.

Nativa, v. 3, n. 1, p. 16-21, 2015. 
MILLER, N. J.; SAMPSON, J.; CANDEIAS, L. P.; BRAMLEY, P. M.; RICE-EVANS, C. A. Antioxidant activities of carotenes and xanthophylls. FEBS Letters, v. 384, n. 3, p. 240242, 1996.

ORDÓÑEZ, J. A. Tecnologia de alimentos: componentes dos alimentos e processos. Vol. I. São Paulo: Artmed, 2005.

PAIVA, M.C.; MANICA I.; FIORAVANÇO J.C.; KIST, H. Caracterização química dos frutos de quatro cultivares e duas seleções de goiabeira. Revista Brasileira de Fruticultura, v. 19, n. 1, p. 57-63, 1997.

PASCUAL-TERESA, S. Molecular mechanisms involved in the cardiovascular and neuroprotective effects of anthocyanins. Archives of Biochemistry and Biophysics, v. 559, n. 1, p. 68-74, 2014.

PATRAS, A.; BRUNTON, N. P.; O'DONNELL, C.; TIWARI, B. K. Effect of thermal processing on anthocyanin stability in foods; mechanisms and kinetics of degradation. Trends in Food Science \& Technology, v. 21, n. 1, p. 3-11, 2010.

PEREIRA, J. M. A. T. K.; OLIVEIRA, K. A. M.; SOARES, N. F. F.; GONÇALVES, M. P. J. C.; PINTO, C. L. O.; FONTES, E. A. F. Avaliação da qualidade físico-química, microbiológica e microscópica de polpas de frutas congeladas comercializadas na cidade de Viçosa-

MG. Alimentos e Nutrição, v. 17, n. 4, p. 437442, 2006.

POIANA, M. A.; MOIGRADEAN, D.; RABA, D.; ALDA, L. M.; POPA, M. The effect of longterm frozen storage on the nutraceutical compounds, antioxidant properties and color indices of different kinds of berries. Journal of

Food, Agriculture and Environment, v. 8, n. 1, p. 54-58, 2010.

PORTELA, I. P.; PEIL, R. N.; RODRIGUES, S.; CARINI, F. Densidade de plantio, crescimento, produtividade e qualidade das frutas de morangueiro "Camino Real" em hidroponia. Revista Brasileira de Fruticultura, v. 34, n. 3, p. 792-798, 2012.

RAFIEIAN-KOPAEI, M.; BAHMANI, M.; KHODADADI, S.; MORADI, M.; KAFESHANI, M. Ameliorative effects of antioxidants on hypertension. Annals of Research in

Antioxidants, v. 1, n. 2, p. 1-6, 2016.

RINALDI, M. M.; COSTA, A. M.; FALEIRO, F. G.; JUNQUEIRA, N. T. V. Post-harvest conservation of Passiflora setacea DC. fruits submitted to different sanitizers and storage temperatures. Brazilian Journal of Food Technology, v. 20, e2016046, 2017.

SALGADO, S. M.; GUERRA, N. B.; MELO FILHO, A. B. Polpa de fruta congelada: efeito do processamento sobre o conteúdo de fibra alimentar. Revista de Nutrição, v. 12, n. 3, p. 303-308, 1999.

SEDIGHI, M.; BAHMANI, M.; ASGARY, S.; BEYRANVAND, F.; RAFIEIAN-KOPAEI, M. A review of plant-based compounds and medicinal plants effective on atherosclerosis. Journal of Research in Medical Sciences, v. 22, n. 30, p. 821, 2017.

SOUZA, V. R.; PEREIRA, P. A. P.; QUEIROZ, F.; BORGES, S. V.; CARNEIRO, J. D. D. S. Determination of bioactive compounds, antioxidant activity and chemical composition of Cerrado Brazilian fruits. Food Chemistry, v. 134, n. 1, p. 381-386, 2012.

SOUZA, V. R.; PEREIRA, P. A. P.; DA SILVA, T. L. T.; DE OLIVEIRA LIMA, L. C.; PIO, R., QUEIROZ, F. Determination of the bioactive compounds, antioxidant activity and chemical composition of Brazilian blackberry, red raspberry, strawberry, blueberry and sweet cherry fruits. Food Chemistry, v. 156, n. 1, p. 362-368, 2014.

SPADA, P. D.; DE SOUZA, G. G. N.; BORTOLINI, G. V.; HENRIQUES, J. A.; SALVADOR, M. Antioxidant, mutagenic, and antimutagenic activity of frozen fruits. Journal of Medicinal Food, v. 11, n. 1, p. 144-151, 2008.

STIPANUK, M. H.; CAUDILL, M. A. Biochemical, Physiological, and Molecular Aspects of Human Nutrition. 3 ed. St. Louis: Elsevier Saunders, 2013.

STRATHEARN, K. E.; YOUSEF, G. G.; GRACE, M. H.; ROY, S. L.; TAMBE, M. A.; FERRUZZI, M. G.; OCHET, J. C. Neuroprotective effects of anthocyanin-and 
proanthocyanidin-rich extracts in cellular models of Parkinson' s disease. Brain Research, v. 1555, n. 1, p. 60-77, 2014.

THREAPLETON, D. E.; GREENWOOD, D. C.; EVANS, C. E.; CLEGHORN, C. L.; NYKJAER, C.; WOODHEAD, C., et al. Dietary fibre intake and risk of cardiovascular disease: systematic review and meta-analysis. British Medical

Journal, v. 347, n. 1, p. f6879, 2013.

VALENCIA, E. F. Los efectos de las antocianinas extraídas de Zea mays L.(Maíz Morado) sobre las hiperlipidemias en ratas albinas. Biotempo, v. 10, n. 1, p. 32-38, 2017.

VANNUCCHI, H.; ROCHA, M. M. Funções plenamente reconhecidas de nutrientes: Ácido ascórbico (Vitamina C). International Life

Sciences Institute, v. 21, n. 1, p. 3-11, 2012.

VEBERIC, R.; STAMPAR, F.; SCHMITZER, V.; CUNJA, V.; ZUPAN, A.; KORON, D.; MIKULIC-PETKOVSEK, $M$. Changes in the contents of anthocyanins and other compounds in blackberry fruits due to freezing and long-term frozen storage. Journal of Agricultural and Food Chemistry, v. 6, n. 29, p. 6926-6935, 2014.

WALLACE, T. C.; GIUSTI, M. M.

Anthocyanins. Advances in Nutrition, v. 6, n. 5, p. 620-622, 2015.

\section{Bruna Aparecida dos Santos \\ Graduanda em Nutrição. Universidade Estadual do Centro-Oeste (UNICENTRO).}

\section{Flávia Teixeira \\ Nutricionista. Mestranda do Programa de Pós- Graduação Interdisciplinar em Desenvolvimento Comunitário da Universidade Estadual do Centro- Oeste (UNICENTRO).}

\section{Luane Aparecida do Amaral}

Nutricionista. Mestranda do Programa de PósGraduação em Saúde e Desenvolvimento na Região Centro-Oeste da Universidade Federal de Mato Grosso do Sul (UFMS).

\section{Gabriela Arelhano Randolpho}

Engenheira de Alimentos. Mestranda do Programa de Pós-Graduação em Saúde e Desenvolvimento na Região Centro-Oeste da Universidade Federal de
Mato Grosso do Sul (UFMS).

\section{Kélin Schwarz}

Nutricionista, Doutora. Docente do Curso de Nutrição da Universidade Federal do Triângulo Mineiro (UFTMG).

\section{Elisvânia Freitas dos Santos}

Nutricionista, Doutora. Docente do Curso de Nutrição e do Programa de Pós-Graduação em Saúde e Desenvolvimento na Região Centro-Oeste da Universidade Federal de Mato Grosso do Sul (UFMS).

\section{Juliano Tadeu Vilela de Resende}

Agrônomo, Doutor. Docente do Curso de Engenharia agronômica e do Programa de PósGraduação em Agronomia da Universidade Estadual do Centro-Oeste (UNICENTRO).

\section{Daiana Novello}

Nutricionista, Doutora. Docente do Curso de Nutrição e do Programa de Pós-Graduação Interdisciplinar em Desenvolvimento Comunitário da Universidade Estadual do Centro-Oeste (UNICENTRO). 